\title{
An unsteady, moving mesh CFD simulation for Harrier hot-gas ingestion control analysis
}

\author{
G. A. Richardson and W. N. Dawes \\ Engineering Department \\ Cambridge University \\ Cambridge, UK
}

\author{
A. M. Savill \\ Computational Aerodynamics Design Group \\ School of Engineering \\ Cranfield University \\ Cranfield, UK
}

\section{ABSTRACT}

Hot gas ingestion (HGI) can be a problematic feature of short take-off vertical landing (STOVL) aircraft during the descent phase of landing, or while on the ground. The hot exhaust gases from the downwards pointing nozzles can be re-ingested into the engine intakes, causing power degradation or reduced engine surge margin. The flow-fields that characterise this phenomenon are complex, with supersonic impinging jets and cross-flows creating large ground vortices and fountain up-wash flows.

A flow solver has been developed to include a suitable linear mesh deformation technique for the descending aircraft configuration. The code has been applied to predict the occurrence of HGI, by simulating experimental results from a $1 / 15$ th scale model of a descending Harrier. This has enabled an understanding of the aerodynamic mechanisms that govern HGI, in terms of the near-field and far-field effects and their impact on the magnitude of temperatures at the engine intake.

This paper presents three sets of CFD results. First a validation exercise shows predicted results from the twin-jet with intake in crossflow test-case. This is an unsteady Reynolds averaged Navier Stokes (URANS) solution for a static geometry (there is no moving mesh). This allows comparison with experiment. Secondly, a full descent phase URANS Spalart-Allmaras (SA) turbulence model calculation is
\end{abstract}

done on an $8.5 \mathrm{~m}$ cell mesh for half the flow domain of the Harrier model and test-rig without dams/strakes. This shows how the HGI flow mechanisms affect the engine intake temperature profiles, for the case where there are no flow control methods on the underside of the aircraft. Thirdly, the full descent phase URANS SA turbulence model calculation is done on a $22.4 \mathrm{~m}$ cell mesh for the full flow domain of the Harrier model and test-rig, with the dam/strake geometry included in the structured mesh region.

\section{NOMENCLATURE}

ADF advanced data format

ATV advanced technology viewer

CFD computational fluid dynamics

DARP Defence Aerospace Research Partnership

DTI Department of Trade and Industry

FOD foreign object debris

hexa hexahedral

HGI hot-gas ingestion

HPCX high performance computer 


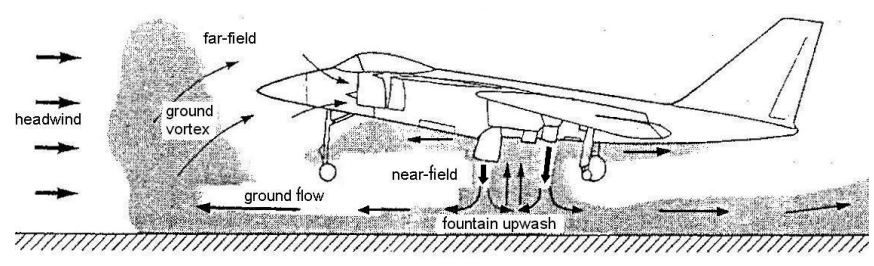

Figure 1. Schematic diagram of hot gas ingestion.

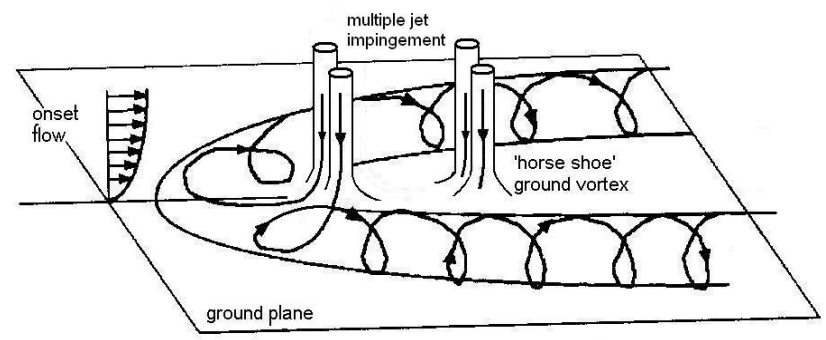

Figure 2. Multiple jet impingement in head wind.

IGES International Graphics Exchange Standard

LES large eddy simulation

OPLUS Oxford Parallel Library for Unstructured Solvers

PDRA Post-Doctoral Research Associate

PUMA Partnership in Unsteady Methods for Aerodynamics

RANS Reynolds averaged Navier-Stokes

SA Spalart-Allmaras turbulence model

STOVL short take-off / vertical landing

tetra tetrahedral

URANS unsteady RANS

VR virtual reality

VRML virtual reality mark-up language

XDB Fieldview (ATV) file format

\subsection{INTRODUCTION}

Some of the background to this work and introduction to HGI is given in Refs 1 and 2. Previously, HGI analysis was done by means of experimental testing in a purpose built test-rig which allows a scale model aircraft to descend in cross-flow, with representative mass-flow and temperatures in the jet plumes. The intake temperatures are measured using thermocouple rakes during the descent. Since the de-commissioning of one such test-rig at Rolls-Royce, it has become more important to replace this capability with a computational method. Hence one of the objectives of this work is to demonstrate that a 'virtual testbed' has been developed, which can be used for future HGI analyses.

The flows below a Harrier are characterised by four high-speed jets impinging on a ground plane. These flows are further complicated by interaction with any cross-flows present, which serve to drive the exhaust flows back towards the descending aircraft. The flow-field contains several components:

- supersonic jet impingement on the ground plane

- fountain upwash flows between the jets

- a low speed head-wind induced 'horse-shoe' vortex

The combination of these effects can cause hot-gas ingestion leading to engine power degradation and/or reduced engine surge margin. Other potential operational problems include:

- variable aircraft pitching moment distributions

- engine ingestion of ground debris (FOD)

- adverse effects on ground crew working environment

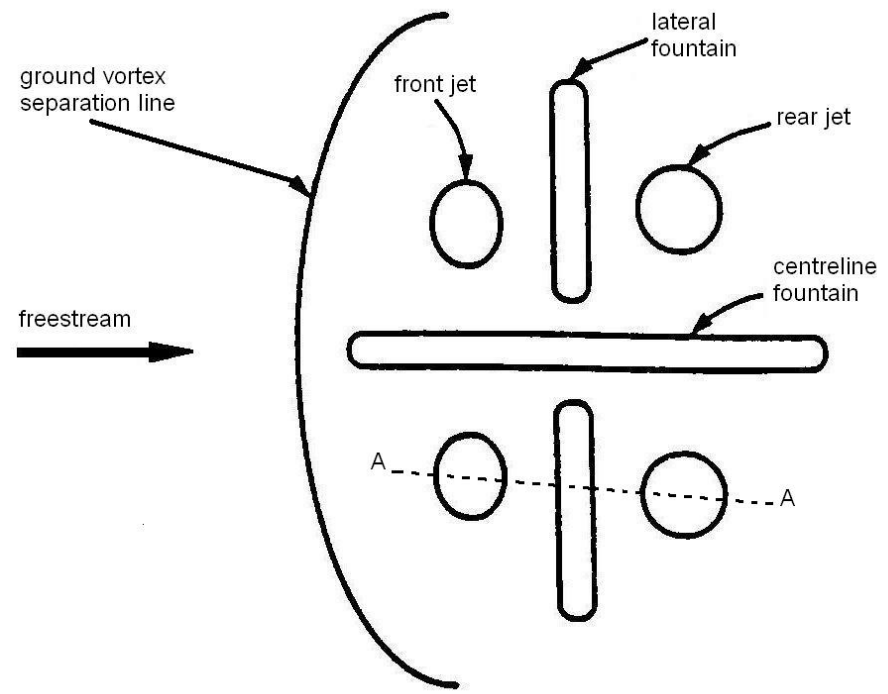

Figure 3. Ground plane jet impingement pattern.

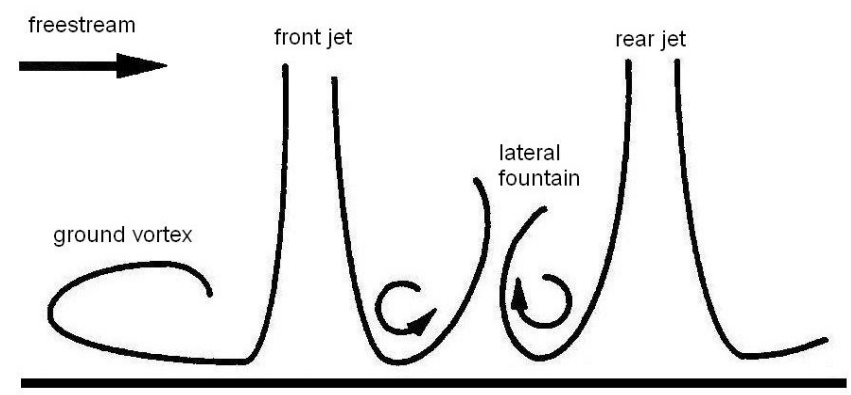

Figure 4. Schematic of HGI flows (section $A-A$ in Fig. 3).

The dominant mechanisms which govern HGI are illustrated in Figs 24. Figure 1, taken from Ref. 3 shows how the influence of HGI can be divided into 'near' and 'far-field' effects. Figure 2 was modified from that given in Ref. 4 and indicates how the hot gases from the nozzles impinge on the ground plane and spread out in all directions. A proportion of the ground plane flow from the front two nozzles extends upstream and encounters the low speed onset boundary layer flow. This causes it to separate and form a 'horse-shoe' shaped ground vortex which rolls-up back towards the aircraft. Some of the far-field flow can be sucked into the intake causing a rise in intake pressure and temperature distortion levels.

'Near-field' effects are dominated by fountain upwash flows caused by the interaction of the exhaust jets with the ground plane (and themselves). The upwash from the impinging jets is forced towards the aircraft fuselage (see Fig. 4) causing localised heating of the aircraft belly and enabling suction from the engine intake to draw these hot gases into the engine. The consequence of these near-field HGI effects can be potentially more severe as the hot-gas takes a shorter path to the intake (compared with far-field flows) and thus undergo less mixing and dilution.

In practice, either mechanism may dominate depending upon factors such as; onset flow direction and strength, cross-flow ratio (ratio of onset flow to jet speed) boundary layer height, aircraft height, fuselage geometry, nozzle configuration, and aircraft descent rate. Broadly speaking, near field effects are more significant closer to the ground (far-field effects being more influential as the aircraft first descends into the hot ground vortex region). The results presented in this paper will show how the fountain upwash flows drive two secondary (nearfield) vortices, immediately below the engine intake, which are thought 


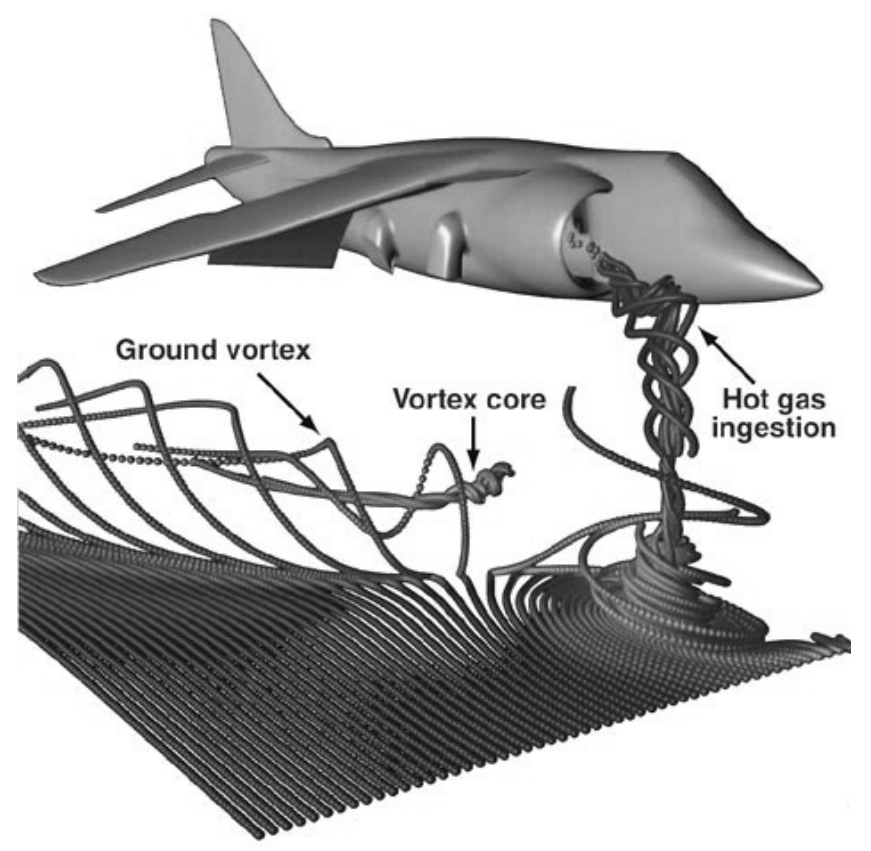

Figure 5. Time-dependent streaklines near ground ${ }^{(6)}$.

to be responsible for the most significant increase in engine intake temperatures.

The scope and contents of this paper are described below. The paper is organised in terms of method, test-case description, results \& discussion, conclusions and future work. The 'method' section describes the full approach taken in terms of mesh generation, code description, solution generation, post-processing, mesh deformation and virtual reality aspects of the project. In the 'test-case descriptions' section, the two test-cases are described. They are referred to as the 'twin-jet with intake in cross flow' test-case, and the 'Harrier model in HGI test rig' test-case. The 'results and discussion' section covers the three solutions which were obtained by modeling these test-cases. The first solution is a $3.3 \mathrm{~m}$ cell unsteady (static mesh) simulation of the twinjet + intake case. The second solution is an $8.5 \mathrm{~m}$ cell (half-domain) unsteady moving mesh solution of the Harrier model descent. The third solution is a $22.4 \mathrm{~m}$ cell (full domain) unsteady moving mesh solution of the Harrier model descent.

\subsection{Literature survey}

A literature survey was conducted at the start of this project in 2004 and papers from the following establishments and authors were studied: Loughborough University, UK (Gary Page, Jim McGuirk, Parviz Behrouzi); NASA Lewis Research Centre, USA (Thomas VanOverbeke, James Holdeman, David Fricker); NASA Ames Research Centre, USA (Merritt Smith, Kalpana Chawla, Neal Chaderjian, Shishir Pandya, William VanDalsem, Thomas Pulliam, Timothy Sandstrom, David Ellsworth). This study found that there had been no published work on the CFD analysis of descending STOVL aircraft. At that time there appeared to be two distinct approaches to the computational analysis of HGI:

1. Static (and descending) jet and intake configurations which represent an aircraft without using the actual surface geometry.

2. Unsteady analysis of static aircraft hovering 'in ground effect', using a realistic representation of the surface geometry.

Loughborough University ${ }^{(5)}$ had done the only descending jet (simplified geometry) analysis and had identified that HGI during

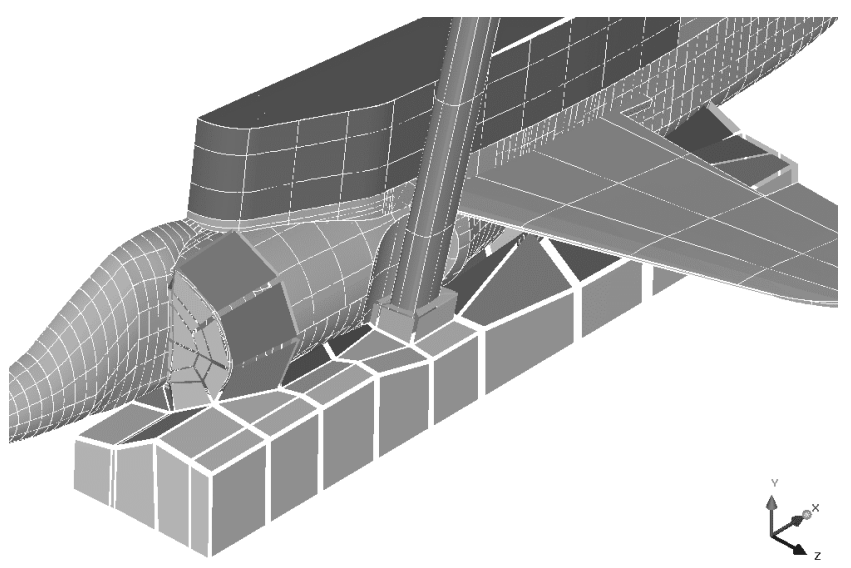

Figure 6. Blocking strategy for a hybrid mesh.

landing is a dynamic event not represented by multiple steady state solutions. They had also done a full (clean) X-32 JSF aircraft modelled for jet-screen analysis, and indicated future intention to look at the descent of full aircraft geometry. They had also indicated that LES is required to compute the unsteady fluctuations of intake temperature rise.

NASA Ames (see Fig. 5) did 35 unsteady simulations of the full Harrier surface geometry at a fixed height (not descent phase). The approach is to produce a parametric database of time-dependent results for various aircraft height and incidence. These have been used to predict characteristics such as hot-gas ingestion, suck-down effect, and ground-cushion effect.

Hence the work reported here is considered to be novel and innovative, given that it explores the capability to model a full aircraft surface geometry (large mesh) during vertical descent, using a URANS moving mesh method.

\subsection{METHOD}

\subsection{Mesh generation}

The aircraft/sting geometry was imported to the ICEMCFD grid generator from a CAD package, in the form of IGES files. The geometry was 'cleaned' and a hybrid mesh created within ICEMCFD, containing a mixture of tetrahedral (or tetra), hexahedral (or hexa) and pyramid volume cells. The mesh was then exported in the form of a FLUENT mesh file. The ICEMCFD package has been installed on a 64-bit machine to enable the memory limitations of 32-bit machines (4GB limit) to be overcome and thus enable larger meshes to be generated.

Considerable effort was put into arriving at a suitable hybrid meshing strategy. It was considered that a hexa mesh was required in a large proportion of the flow domain. It is generally accepted that a tetra mesh is more dissipative than a hexa mesh of similar size (in terms of cell count). It is important to avoid too much diffusion of the jet plumes and fountain upwash flows, which would arise if a tetra mesh were used in this region. The use of a hexa mesh ensures that the cell faces are aligned with the flow direction, and hence numerical diffusion is minimised.

Also for this application, a tetra mesh is not considered suitable for LES calculations because it is more important to avoid rapid changes in the mesh density. Hence a tetra mesh was retained for the less important regions around the aircraft geometry which are difficult to mesh using a structured approach.

Figure 6 shows the structured mesh faces at the interface between the tetra/hexa mesh regions. The hexa mesh was applied for most of 


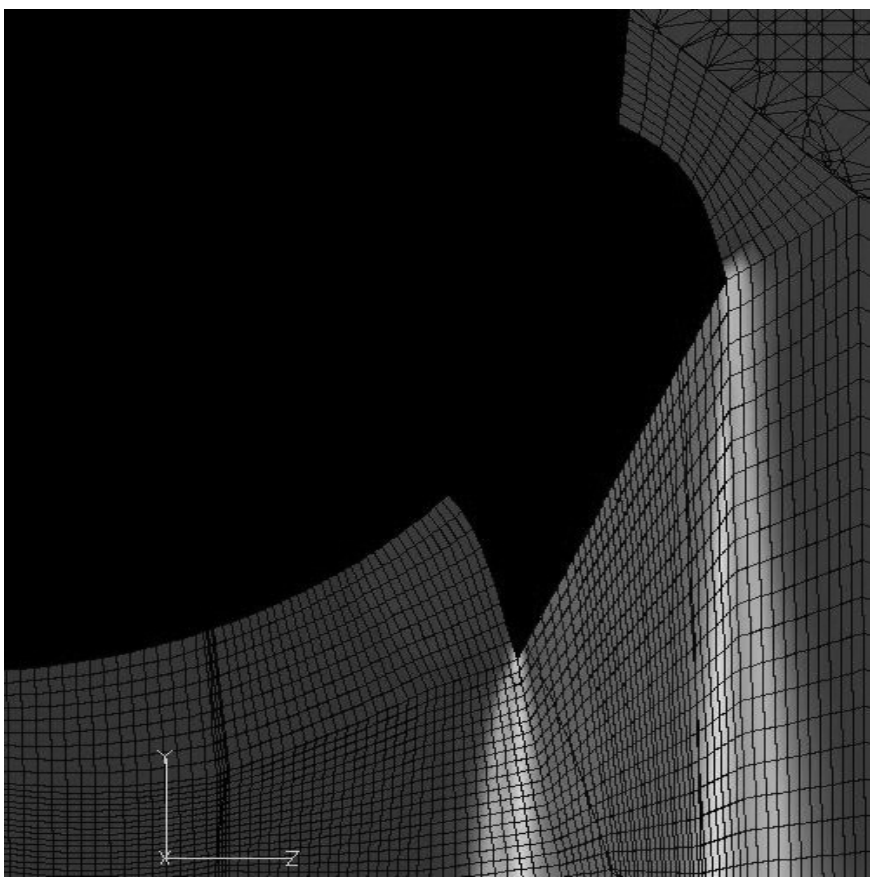

Figure 7. Mesh density around aft nozzle exit.

the flow domain, including the area along the aircraft belly, and underneath the exit planes of the four nozzles. A hexa mesh was also applied in the engine main intake and auxiliary intakes. In particular it was considered important to use hexa mesh to resolve the four jet plumes, the ground vortex, the fountain upwash flows, and the flows along the underside of the aircraft. One draw-back of the hybrid meshing approach is that it can be very time consuming to generate meshes on complex geometries. Figure 7 shows the degree of mesh refinement used at the nozzle exit planes. $\mathrm{O}$ grids were used to capture the geometry of the jet nozzles both inside and outside the jet plume region. The nozzle internal flow was not modelled - only flow downstream of the nozzle exit planes was predicted.

\subsection{Code description}

The HYDRA code is a suite of non-linear, linear and adjoint CFD solvers developed collaboratively by Rolls-Royce and its University partners. It is a general purpose code for hybrid unstructured meshes which uses an efficient edge-based data structure by Moinier and Giles $^{(7)}$. The flow equations are integrated around median-dual control volumes using a MUSCL (monotonic upwind scheme for conservation laws) based flux-differencing algorithm. Turbulence is modelled using the either the Spalart-Allmaras ${ }^{(8)}$ or $k-\varepsilon$ turbulence models. A Smagorinsky based LES capability is also available. The discrete flow equations are preconditioned using a block Jacobi preconditioner $^{(7)}$ and iterated towards steady state using the five-stage Runge-Kutta scheme of Martinelli ${ }^{(9)}$. Convergence to steady state is further accelerated through the use of an element-collapsing multigrid algorithm ${ }^{(10)}$. Time-accurate solutions are obtained using either implicit dual time-stepping, with preconditioning and multi-grid on the inner steps, or with explicit time-stepping. Mixing and sliding plane capabilities are available for multistage turbo-machinery. The flow solver has been parallelised using the domain decomposition method and runs efficiently on both shared and distributed memory computers.

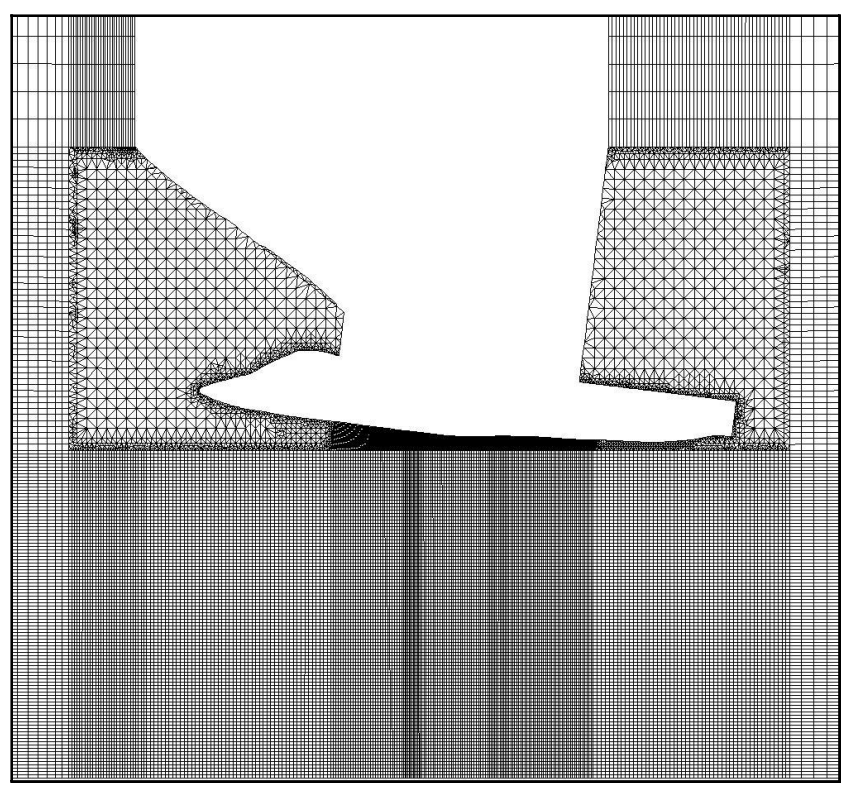

Figure 8. Mesh deformation at start of descent.

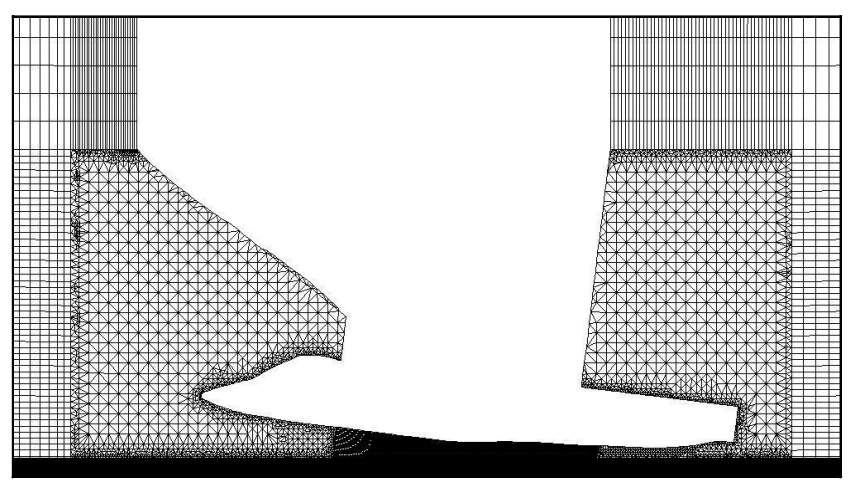

Figure 9. Mesh deformation at end of descent.

\subsection{Solution generation}

The solver is installed on the HPCx IBM 64-bit supercomputer allowing it to be run in parallel on a large number of processors. The 'full domain' Harrier (run 39) took about 20 separate runs to complete the unsteady descent phase, using 256 processors for 12 hours at a time. On completion of the solution, typically 100 unsteady solution files are created, each containing current (deformed) grid and flow-field data.

\subsection{Mesh deformation}

The pre-existing moving mesh capability within the solver was as described in Ref. 11. The present project has further developed this to allow 3D mesh deformation for a full aircraft in descent. A detailed description of the linear mesh movement algorithm, which has been added to the code, is described in Ref. 1. For translational mesh movement the mesh translates at a constant rate from its initial position to a final position defined by the perturbations in the mesh movement file. The user prescribes the times (in seconds) at which the movement begins and ends. These allow the mesh to remain in a static position either before and/or after the mesh translation. Figures 8 and 9 demonstrate the mesh deformation at the aircraft centre-line plane. In this case the grid nodes move only in the vertical 


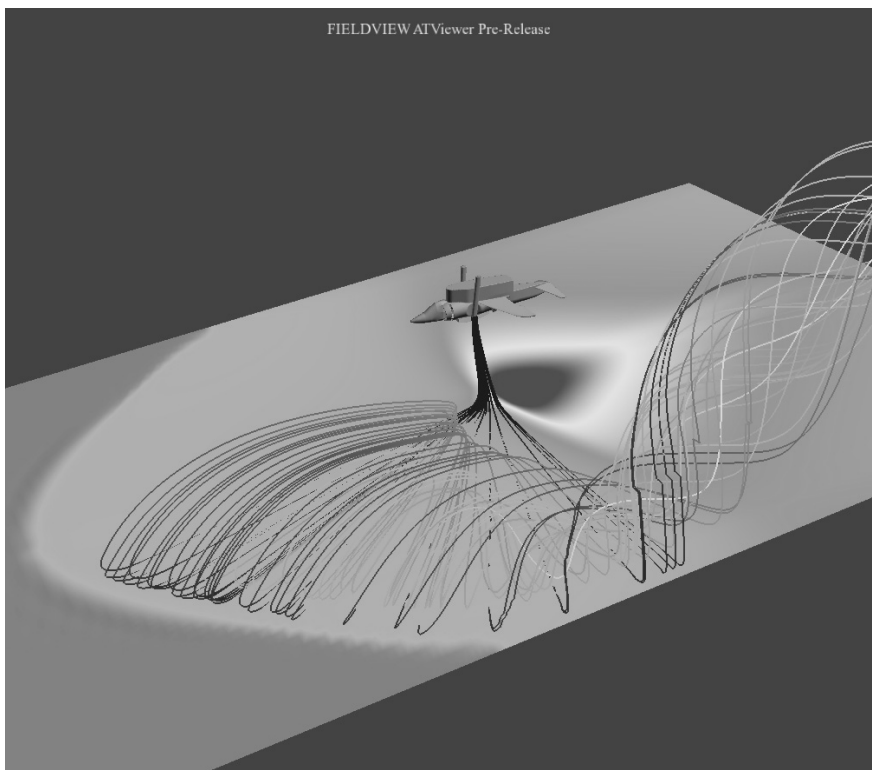

Figure 10. Sample output from stereo ATV pre-release.

$y$-direction. It can be seen that there is a square region of unstructured mesh immediately surrounding the aircraft, outside which the mesh is fully structured. Figure 8 shows the starting mesh which represents the aircraft in hover. Figure 9 represents the mesh as the aircraft lands on the ground plane. This demonstrates how the structured mesh below the aircraft becomes compressed as the model descends and approaches touch-down. For this reason the undercarriage is not included in the analysis, since there should be a clear gap between aircraft and ground, on landing. If the mesh did include any geometry in this region, it would become distorted during the descent phase of the simulation.

\subsection{Post processing}

Virtual reality techniques have been used to visualise the ground vortex flows around the Harrier during the descent phase. This has been achieved using the Fieldview Advanced Technology Viewer (ATV) software which is compatible with a Polaroid twin projector system. Figure 10 shows how the output from Fieldview ATV package, is being used to view the front jet pathlines and ground plane temperatures while the aircraft model is in hover.

\subsection{TEST-CASE DESCRIPTIONS}

\subsection{Twin-jet with intake in cross-flow}

\subsubsection{Experimental conditions}

A twin-jet with intake in cross-flow study has been used to validate the hybrid meshing approach that we have used for the Harrier model analysis. Figure 11 shows the geometry and flow domain. This was used as a 'building block' test-case, to compare our RANS results against existing experimental data. Furthermore these results and experimental data are compared with LES results (on the same mesh) ${ }^{(12)}$

The experimental study by Behrouzi and $\mathrm{McGuirk}^{(3)}$ was done in a water tunnel using LDA specifically to provide validation data for CFD. The mass flow through the intake is equal to that of the two

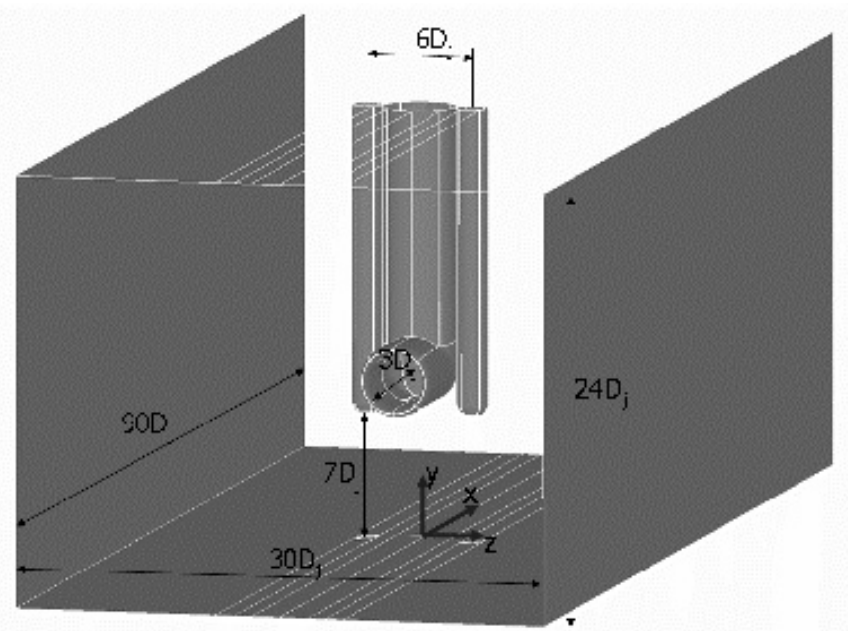

Figure 11. Twin-jet with intake geometry.

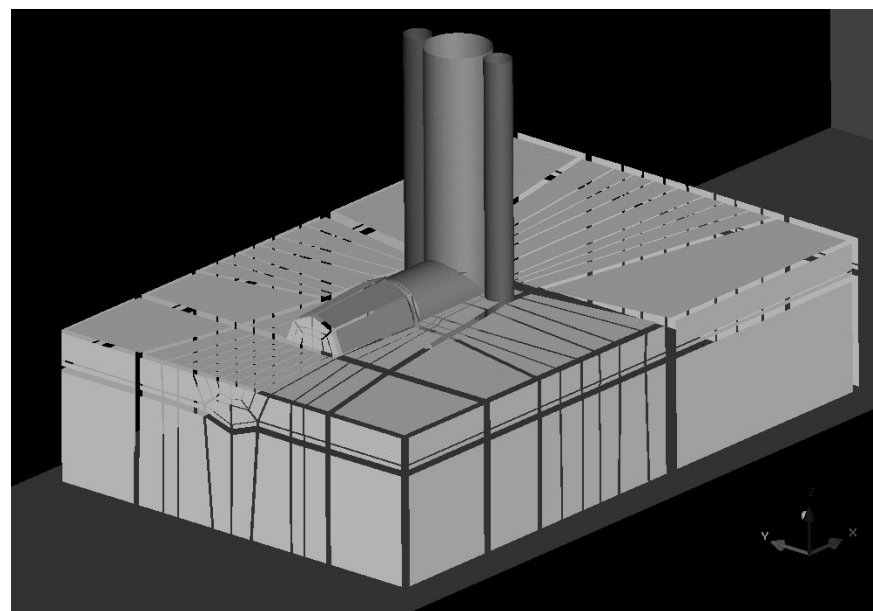

Figure 12. Tet/hex interface plane.

jets. The location of the intake in relation to the jet nozzles is similar to that of the Harrier. The jet to cross-flow ratio is 24 and the impingement height of the jets is $7 D_{j}$. The jet Reynolds number based on nozzle diameter is 37,500 . The size of $D_{j}$ is $0.0125 \mathrm{~m}$ in the experiment. For the CFD analysis of this test-case, the mesh was created using ICEMCFD and contains $3.3 \mathrm{~m}$ cells and $1.8 \mathrm{~m}$ nodes.

\subsubsection{Computational set-up}

In the CFD analysis the geometry is scaled such that $D_{j}=0.00471 \mathrm{~m}$ in order to keep the Reynolds number $\operatorname{Re} j=40,000$. The jet velocity is set to $V_{j}=120 \mathrm{~ms}^{-1}$ at a location $0 \cdot 25 D_{j}$ downstream of nozzle exit plane. The intake velocity is set to $V_{i}=27 \mathrm{~ms}^{-1}$ (based on area ratio and mass-flow). A cross-flow velocity $V_{c}=5 \mathrm{~ms}^{-1}$ is set by applying a total pressure $P o=101,345 \mathrm{~Pa}$ at the upstream boundary, and a static pressure $p=101,325 \mathrm{~Pa}$ at the downstream boundary. The side walls were set as inviscid wall boundaries and the top/bottom walls were set as viscous wall boundaries. All surfaces associated with the model geometry are modelled as viscous walls. The SpalartAllmaras one-equation turbulence model option was applied. Figure 12 shows the interface plane of the hex blocking structure used. 


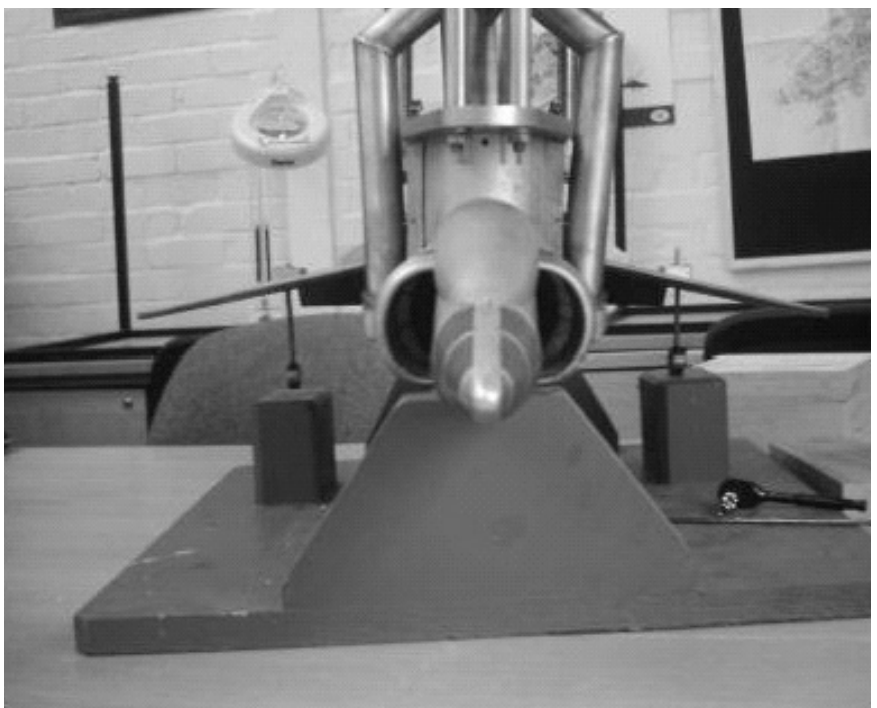

Figure 13. Photo of Harrier test rig model.

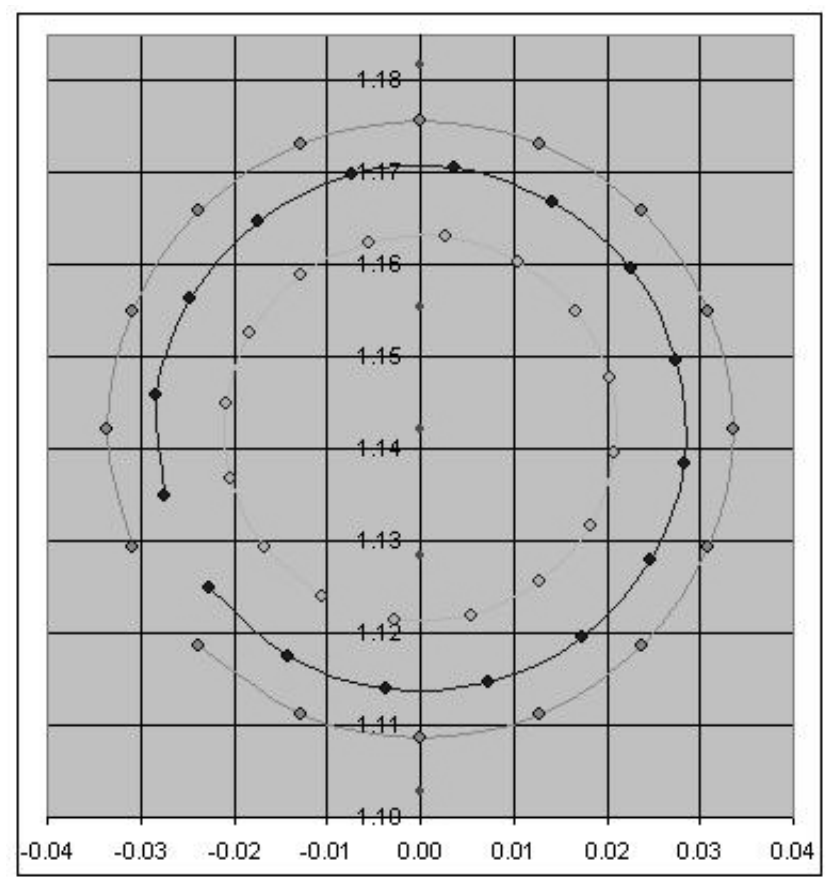

Figure 14. Test-rig engine intake thermocouple locations.

\subsection{Harrier model in HGI test-rig}

\subsubsection{Experimental conditions}

A complete description of the experimental details can be found in Refs 13 and 14. The tests were carried out on a 1/15th scale geometrically representative model of the AV-8B aircraft fitted to a VTOL rig inside a low speed wind tunnel. The aircraft model was set at $7.5^{\circ}$ nose-up incidence with the auxiliary doors open. The production standard deep strake and dam geometry (without gun-pods) was used and variations on the dam/strake geometry were also tested. The model was tested under vertical landing and 'no-go VTO' (committed touch-down) conditions, for a range of headwinds. The

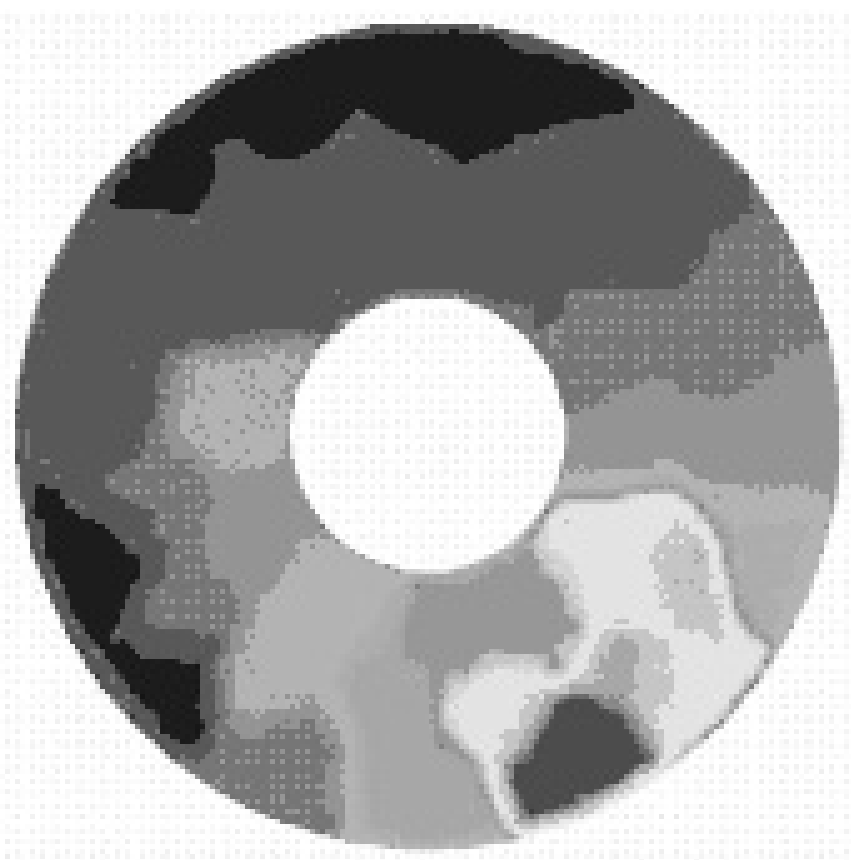

Figure 15. Experimental temperature contours interpolated from engine intake thermocouple rakes, where the temperature range is $15^{\circ}$ to $35^{\circ}$ deg $\mathrm{C}$.

tests were all carried out at full-scale pressures, and at temperatures less than full scale due to a rig limit of $750 \mathrm{~K}$. Repeat runs were performed for every configuration to allow averaging of the results in view of the unsteady nature of the HGI process. Figure 13 shows the Harrier test-rig model and Fig. 14 shows the locations of the Harrier model intake thermocouple rakes.

The Harrier model and HGI test-rig were not instrumented with a view to CFD validation, therefore only predicted engine face temperatures could be compared with test-data. During testing, the engine intake of the aircraft test-piece contained three annular thermocouple rakes to record engine face transient temperatures. Each rake has 16 fast response thermocouple probes to measure the intake temperatures during the aircraft descent. The test-data is available as engine intake temperature profiles against elapsed descent time.

The experimental engine intake temperature profiles were animated to interpret which regions of the intake area correspond to the greatest temperature increase. Figure 15 shows a snapshot taken from the last frame of this animation. The asymmetry shown here may reflect the asymmetry of the experimental test-rig and wind tunnel geometry.

\subsubsection{Computational set-up}

The computational set-up matches the experimental conditions. The Harrier model descends a distance of $0.446 \mathrm{~m}$ at a rate of $1 \mathrm{~ms}^{-1}$ in a low speed head wind of $6 \mathrm{~ms}^{-1}$. The initial engine intake flow conditions are Ttotal $=295 \mathrm{~K}$, mass-flow $=0.445 \mathrm{kgs}^{-1}$. Boundary conditions corresponding to nozzle pressure ratios of 2.5 and 2.0 were applied at the exit planes of the front and rear nozzles, respectively.

For the 'half-domain' run, only one half of the flow domain is meshed and a symmetry boundary is applied at the aircraft centreline $(x-y$ plane). The solution is based on a hybrid mesh with 8.5 million cells and $6 \mathrm{~m}$ nodes. The structured block extends down from the nozzle exit planes to the main structured blocks that surround the aircraft. The structured mesh also extends down from the aircraft 


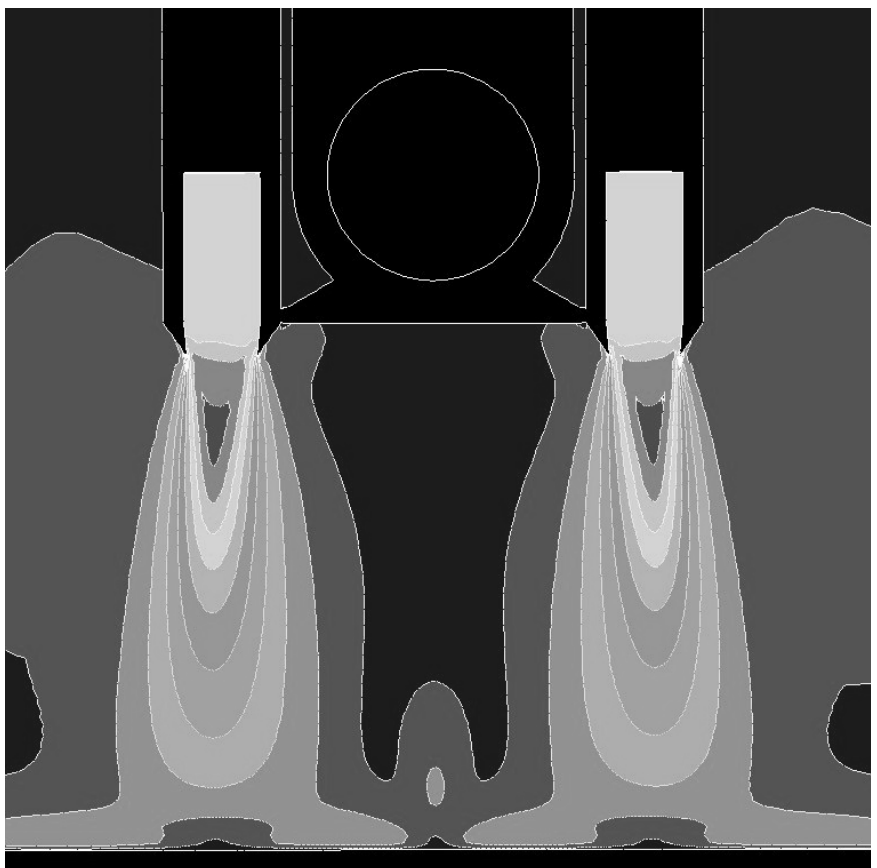

Figure 16. Mach contours $(0 \cdot 0-0 \cdot 4)$ at nozzle plane.

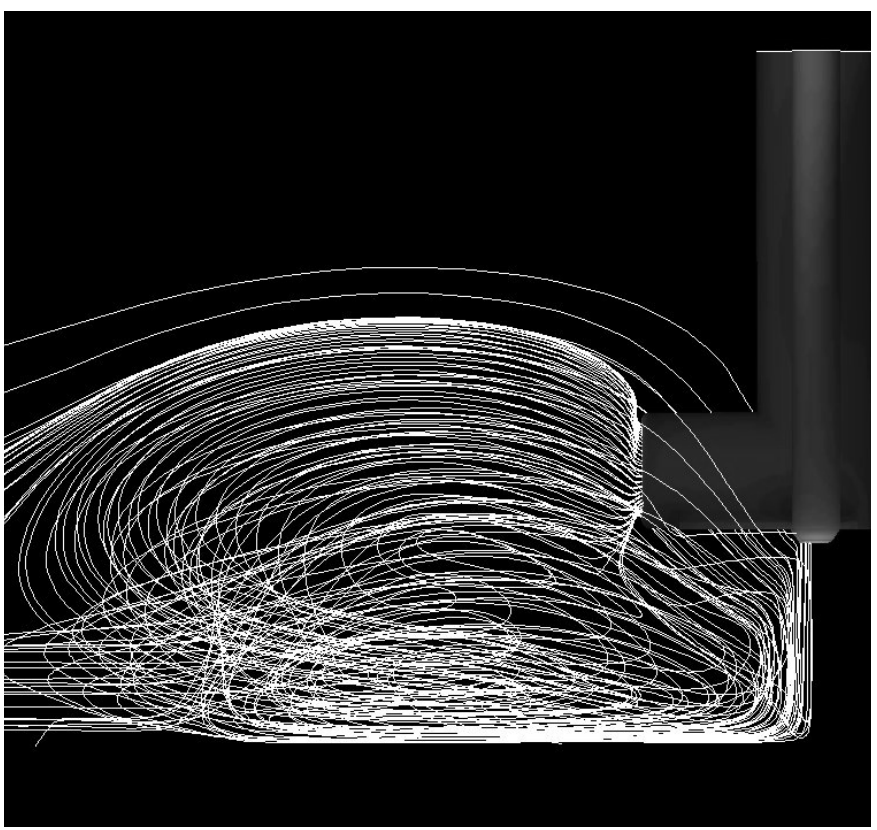

Figure 17. Reverse flow pathlines from intake.

underside. There is no structured mesh at the engine intake and the auxiliary intakes are included as tetra mesh regions. The dual timestepping implicit solver option was used, and the time-step size was $0.001 \mathrm{sec}$ (equivalent to $1 \mathrm{~mm}$ of vertical descent). Only ten iterations were applied at every time-step in order that the full vertical descent may be achieved in one single 12 hour run on HPCx, based on the initial flow solution.

For the 'full-domain' run, half of the flow domain is meshed and this mesh is then copied across the centreline plane, to create a mesh for the full-domain. The full domain was meshed (despite the antici-

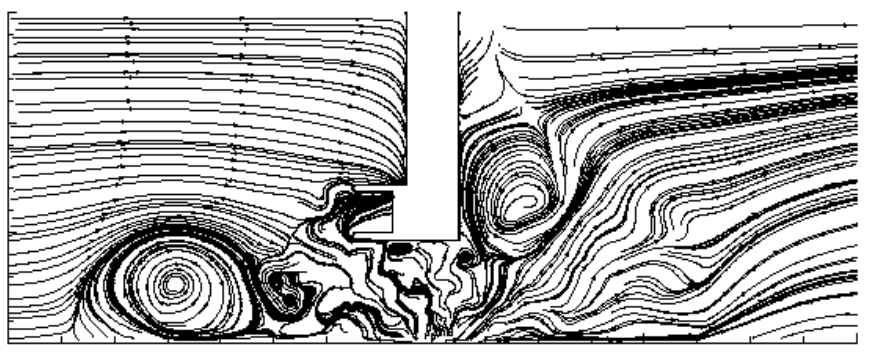

Figure 18. Streamlines from LES solution ${ }^{(12)}$.

pated symmetrical URANS solution) to allow for and capture any unsteady asymmetrical effects. Such effects are more likely to occur with a LES analysis. The full domain mesh and unsteady solution was required for use as initial conditions for the LES analysis reported in Ref. 12 .

The solution is based on a hybrid mesh with $22.4 \mathrm{~m}$ cells and $17.3 \mathrm{~m}$ nodes. The hybrid mesh strategy is similar to the halfdomain run but in this case hexa mesh regions cover more of the flow domain. The hexa mesh extends all the way around the aircraft underside, up to and above the nozzle exit planes. There are 33 cells across the aft nozzle exit plane (as shown in Fig. 7), and a similar level of mesh refinement is applied at the forward nozzle.

The standard dam/strake configuration geometry is included within the hexa mesh region. The intake region and auxiliary intakes are resolved with structured mesh, which extends upstream from the intake leading edge. The near wall mesh size on the ground plane is $1 \mathrm{~mm}$, which will become smaller as the aircraft descends and the mesh there becomes compressed. The dual timestepping implicit solver option was used, and the time-step size was $0.001 \mathrm{sec}$ (the same as 'half-domain' run), but in this case 100 iterations were applied at every time-step. Only the ground plane is set as a viscous wall boundary condition, with all other model geometry being specified as inviscid walls. The total simulation time is $1.0 \mathrm{sec}$, with the aircraft model descending for the initial $0.445 \mathrm{sec}$, and then static on the ground for the remaining $0.555 \mathrm{sec}$.

\subsection{RESULTS AND DISCUSSION}

\subsection{Twin-jet with intake in cross-flow}

Mach contours at the nozzle centerline plane are shown in Fig. 16. The potential cores of the jet flows are too short, since the shear layers appear to grow too rapidly. The potential core length (where the centreline velocity remains unchanged) should be between 8$12 \mathrm{Dj}$. The static pressure at impingement should be equal to the jet total pressure $P_{0}$. However, in this case, this rapid decay of the jet flows means a loss of total pressure in the jets and the jet centerline velocity is reduced by roughly $50 \%$ near to the ground plane. This is due to over-prediction of eddy-viscosity in the SA turbulence model. This problem can be overcome by modifying the SA model as described in Ref. 4, but this was not achievable within the time constraints of this project.

In Fig. 17, reverse flow pathlines from the engine intake boundary show the ground vortex recirculation upstream of the intake. The fountain upwash flows can also be seen to enter the bottom centre region of the intake geometry. Figure 18 shows the flow streamlines gained from an LES solution. It can be seen here that the URANS solution is quite different from the LES one, in terms of the size/location of the ground vortex. 


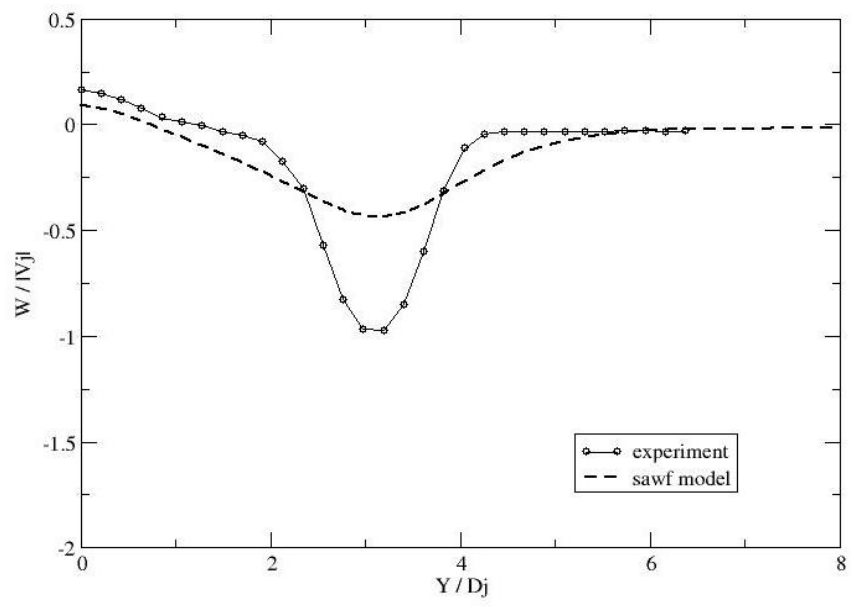

Figure 19. Mean vertical velocity across jet and fountain ( $2 D_{j}$ from ground).

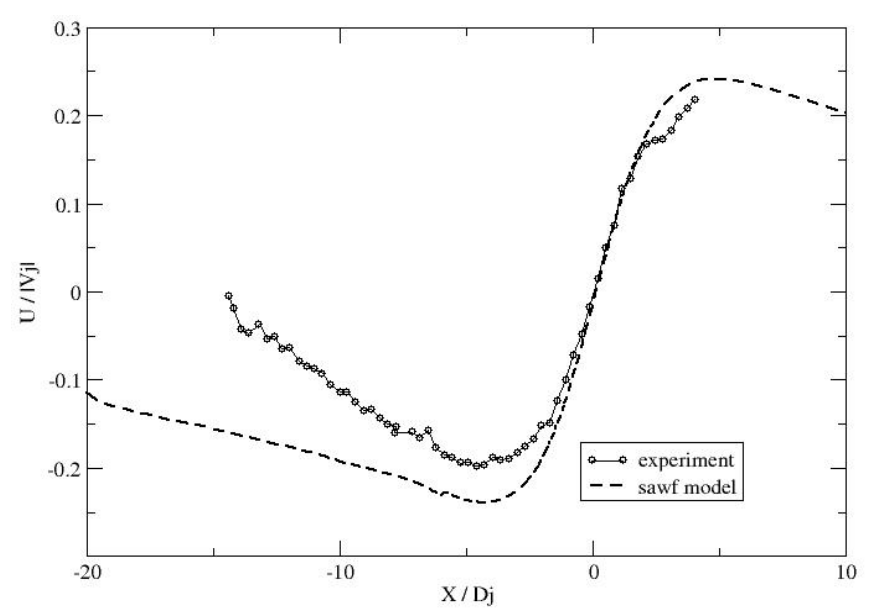

Figure 20. Streamwise velocity on symmetry plane $\left(0.25 D_{j}\right.$ from ground).

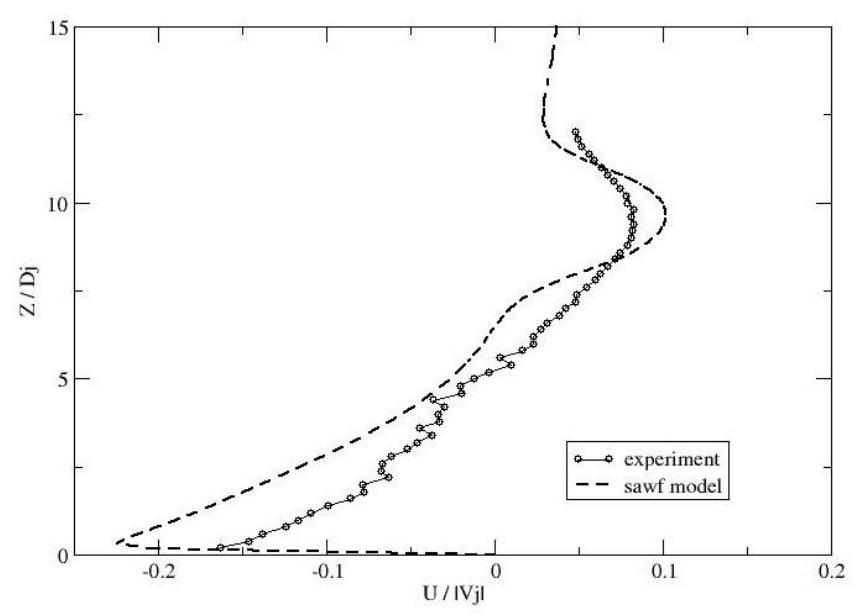

Figure 21. Streamwise velocity on symmetry plane $\left(1 D_{j}\right.$ ahead of intake).

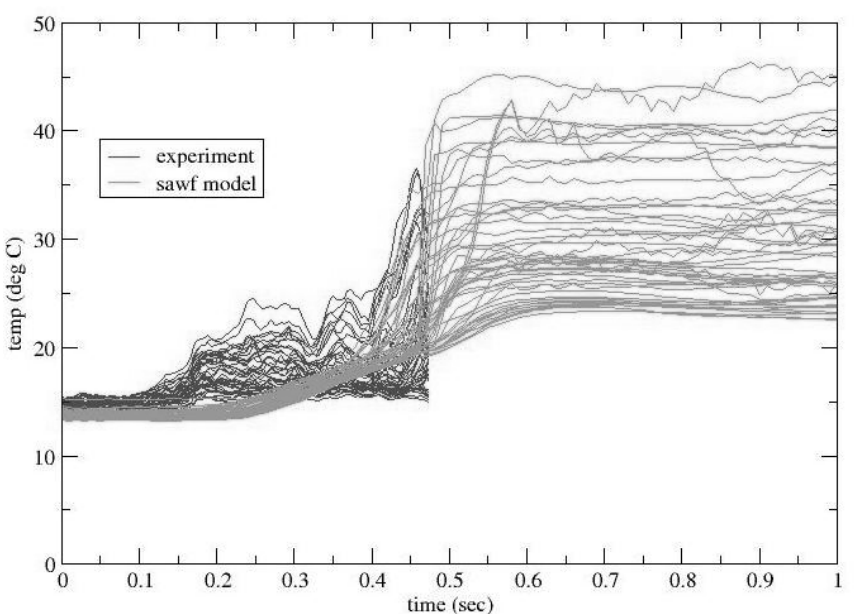

Figure 22. Intake temperature profiles.

Figures 19 to 21 show comparisons of predicted velocity profiles with experimental data for the twin-jet with intake test-case. Equivalent results for RANS $k-\varepsilon$ and LES predictions for this testcase may also be found in Ref. 12. Figure 19 shows the vertical velocity under the jets, $2 D_{j}$ from the ground plane, taken in a spanwise direction underneath the jet centerlines. This shows how much the predicted jet flows have decayed compared with the experimental data. The vertical velocity either side of the jet centerline is over-predicted compared with experiment. The magnitude of fountain upwash flows is also under-predicted. Figure 20 shows the stream-wise velocity component at the symmetry plane, $0 \cdot 25 D_{j}$ from the ground plane, taken in a stream-wise direction. This shows that the stream-wise extent of the ground vortex upstream is being overpredicted. Figure 21 shows the stream-wise velocity component $1 D_{j}$ ahead of the intake, taken in a vertical direction. This confirms what is being shown in Fig. 20 since the velocity magnitude in the ground vortex region is being over predicted. These results indicate that the key flow features (i.e. fountain upwash flows and ground vortex) are being predicted. However the detailed comparison with experiment is poorer due to the over prediction of eddy viscosity in the jet shear layers by the turbulence modelling.

\subsection{Harrier model - half domain}

The results from the 'half-domain' case have been described in more detail in Ref. 2. This run was for only one half the flow domain, and did not include the dam/strake geometry on the aircraft model. Also, the extent of the structured mesh is more limited compared with the 'full-domain' run. The area around the intake and auxiliary intakes was covered by tetra mesh here.

Reference 2 includes discussion of how the predicted engine intake temperature profiles can be explained by studying the HGI flow mechanisms. Briefly, this is described as follows: as the aircraft starts to descend the intake temperatures remain constant since the model is outside of any hot-gas regions; as the engine intake becomes engulfed in the warm re-circulating air of the far-field ground vortex, the initial rise in engine intake profile temperatures is seen; as the aircraft model descends further it begins to suck in the hotter flows from the near field (fountain up-wash driven) ground vortex; this causes a sudden increase in the intake temperatures since the near-field flows are less well mixed (less cooling) compared to the far-field flows. These CFD results show that mean engine intake temperatures continue to rise quite rapidly as the aircraft approaches touchdown, however the experimental results do not continue after the touchdown has occurred. 


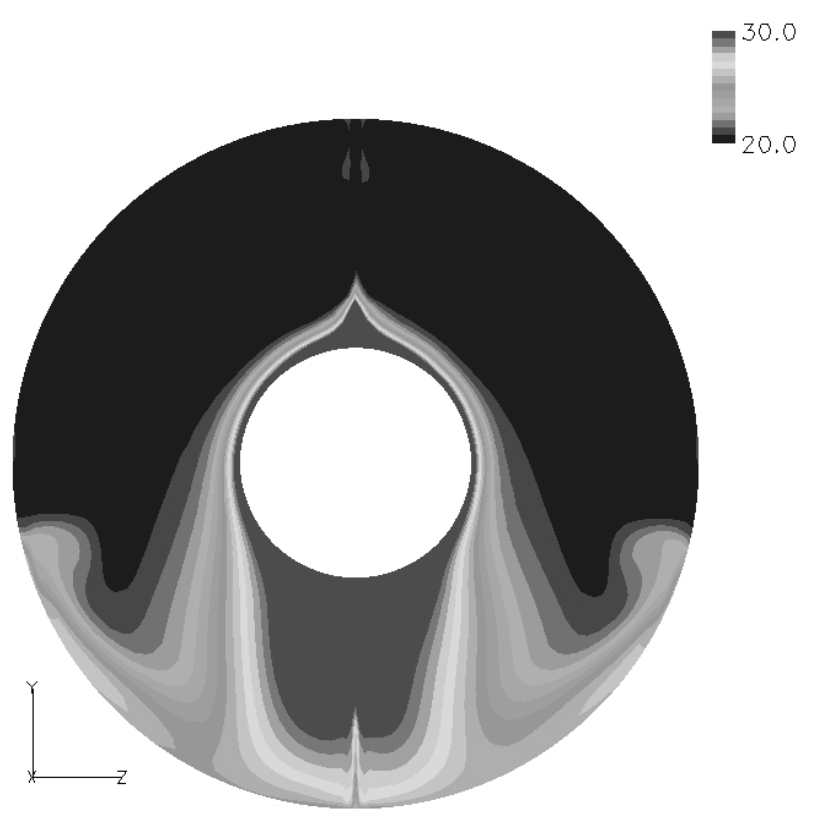

Figure 23. Intake temperature contours $\left({ }^{\circ} \mathrm{C}\right)$.

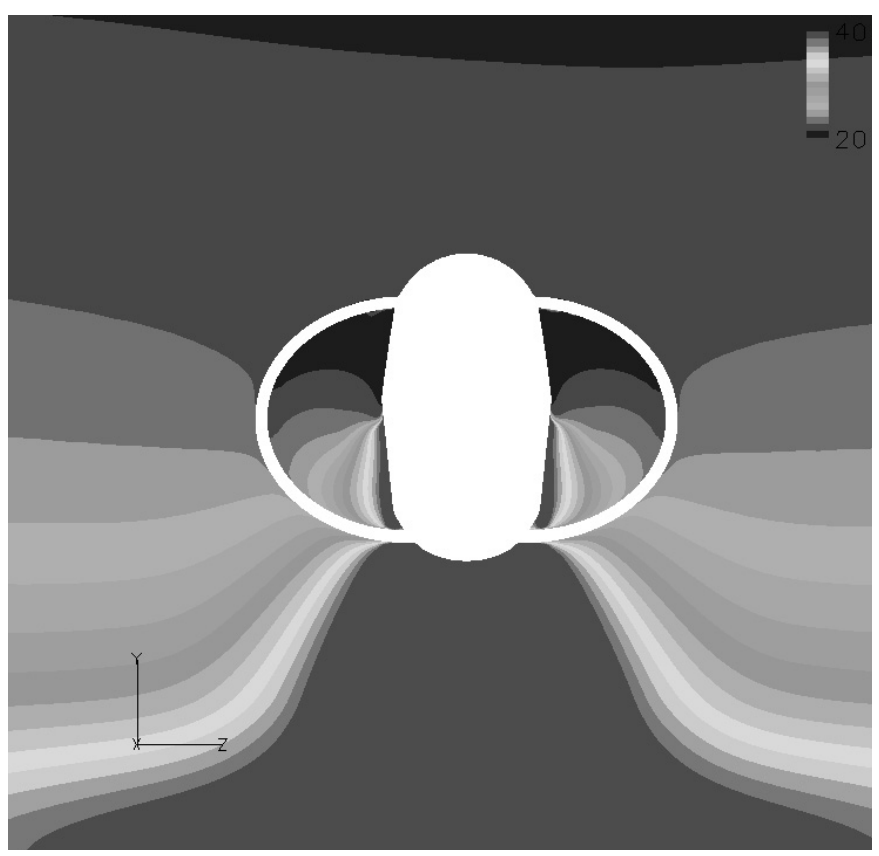

Figure 24. Intake temperature contours $\left({ }^{\circ} \mathrm{C}\right)$.

\subsection{Harrier model - full domain}

VR techniques were used (Fig. 10) to visualise the exhaust plume flows in 3D to gain a better understanding of the flowfield in terms of near-field and far-field effects.

A comparison of experimental and predicted engine intake temperature profiles is shown in Fig. 22. Broadly speaking this shows that the intake temperatures due to the far-field ground vortex region are under-predicted, whereas the intake temperatures due to the near-field fountain upwash flows are being predicted much better. This also shows that the peak temperatures in the engine intake at the end of the descent $(0 \cdot 4-0.445 \mathrm{sec})$ are being predicted

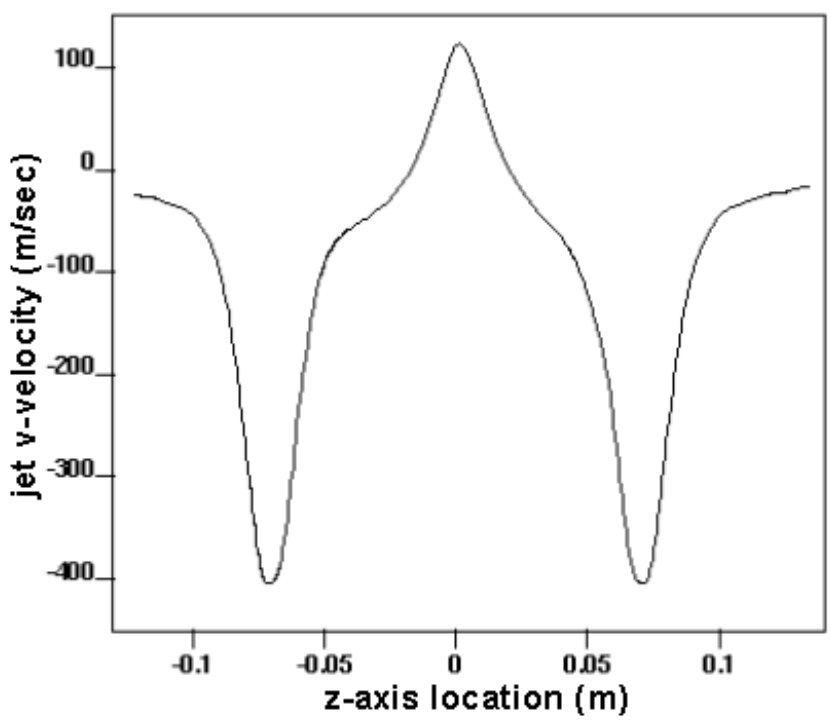

Figure 25. V-velocity profile under jets.

well, although this does not mean that temperature variation with location is well predicted.

In Fig. 23 the predicted engine intake (flow outlet boundary) temperature contours are shown in the range $20-30^{\circ} \mathrm{C}$. This corresponds to a simulation time of $0.445 \mathrm{sec}$, when the aircraft model has just finished the descent phase. This figure can be directly compared with the experimental results shown in Fig. 15. The predicted results show similar peak intake temperatures compared with experiment. It is clear that the predicted results are more symmetrical than experimental ones.

The engine intake temperature contours are shown in Fig. 24, at a cut-plane level with the leading edge of the intake. The peak temperatures in this plot show how the fountain upwash flows are directed to the bottom side of the intake and then split either side of the aircraft nose. The results shown in Figs 23 and 24 indicate that the temperature distribution changes significantly between the leading edge of the intake, and further downstream at the engine face. There is a change of the intake cross-section as the two halves of the intake (at the leading edge) converge towards the circular section at the engine face.

In Fig. 25 the fountain upwash ( $v$-velocity) profiles underneath the front nozzles are shown. This profile was taken at time $t=1.0 \mathrm{sec}$ and at a location which is roughly half way between the nozzle exit planes and the ground plane. This predicted data gives an impression of the magnitude of fountain upwash flows compared with the jet velocity. The peak predicted fountain upwash flow was found to be around $150 \mathrm{~m} / \mathrm{sec}$. This velocity profile also shows some asymmetry.

Ground vortex pathlines at time $t=0 \cdot 0 \mathrm{sec}$ when the model simulates hover (Fig. 26) and time $t=1 \cdot 0 \mathrm{sec}$ after it has landed (Fig. 27) are shown. The pathlines are released from a location at $0.001 \mathrm{~m}$ from the ground, upstream of the model. The initial pathline pattern shows a large ground vortex which extends in a parabolic shape (from a plan view) upstream of the aircraft. The pathlines are in line with the higher temperatures shown on the ground plane. An animation of all the pathline predictions (from 100 unsteady flow solutions) was created. This showed that as soon as the aircraft starts to descend, the ground vortex recedes (downstream) and appears to collapse. After the aircraft has landed (time $t=0.445 \mathrm{sec}$ ) the flowfield is still changing, but the unsteady behaviour of the ground vortex appears to have mostly finished at the time $t=1 \cdot 0 \mathrm{sec}$. At this time there appears to be two smaller ground vortex-like flow structures upstream of the aircraft model - as indicated by the higher ground plane temperatures in that region. 


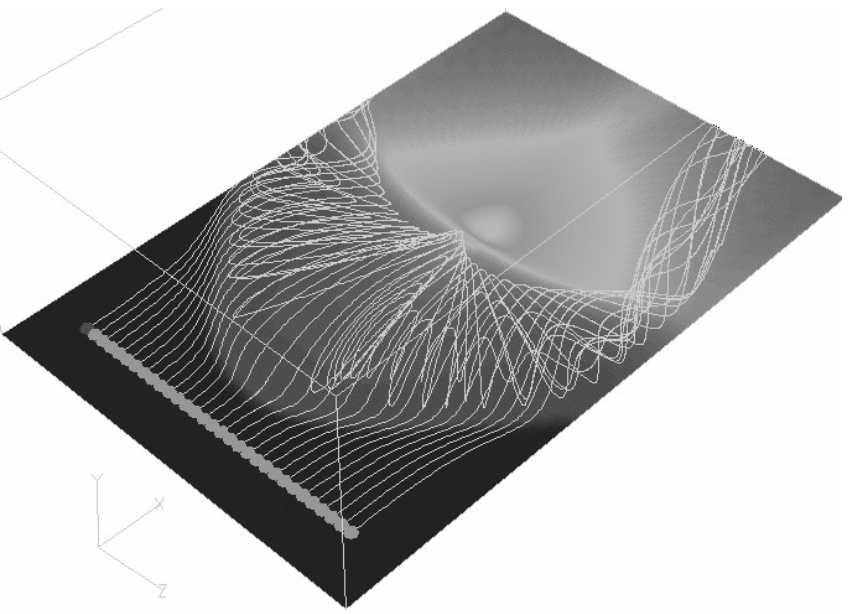

Figure 26. Pathlines at start time $t=0 \cdot 0$.

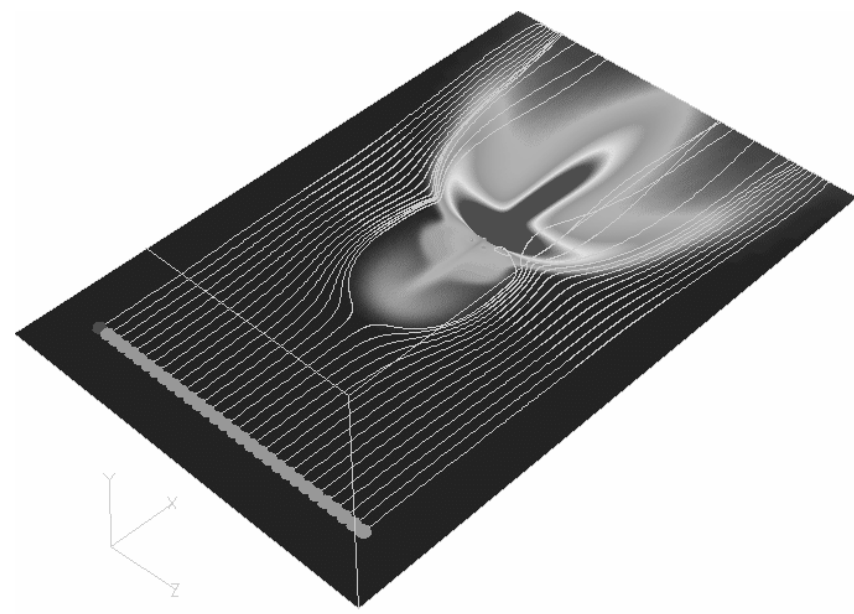

Figure 27. Pathlines at finish time $t=1 \cdot 0$.

In Fig. 28 the ground plane temperature contour plots are shown, with finer contour levels to Fig. 27, after the aircraft has landed. The peak static temperatures below the front and rear nozzles are of interest here. The peak temperature on the ground below the front nozzle is equal to the value of the front nozzle inlet total temperature, $T_{0}$. This plot also shows the distinction between the regions of the ground plane which are heated by the front and rear nozzles. This shows that the ingested flows (for this type of low speed headwind vertical descent case) will always be dominated by the flow from the front nozzles. The theoretical absolute maximum temperature at the intake will be the front nozzle $T_{0}$. Due to mixing of the front nozzle jet flows prior to entering the intake, the ingested flow temperatures are significantly less than $T_{0}$.

The temperature contours at a vertical section which cuts through part of the intake and the dam are shown in Figs 29 and 30. These views are useful to analyse the effect that the dam/strake geometry has on the HGI process. Figure 29 taken just before landing shows the higher temperature fountain upwash flows being held back by the dam, while the cooler far-field flows are being ingested. Figure 30 taken at time $t=1 \cdot 0 \mathrm{sec}$, shows that the fountain upwash flows have spilled over the dam and entered

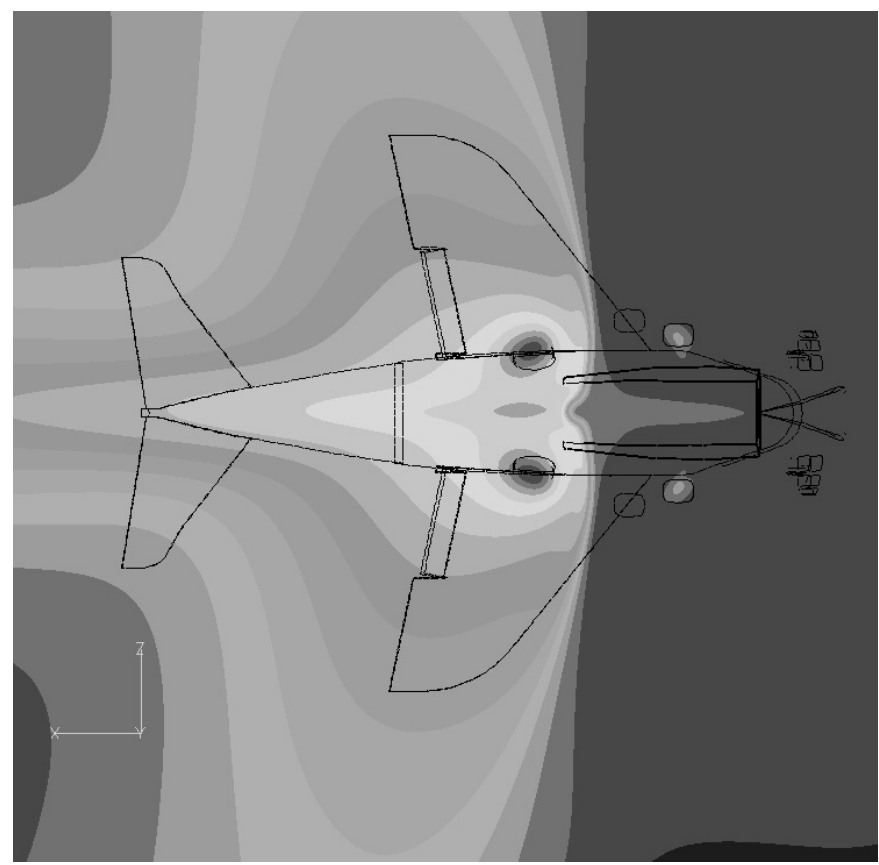

Figure 28. Ground plane temperature contours in the range $288-650 \mathrm{~K}$, at time $t=1.0 \mathrm{sec}$.

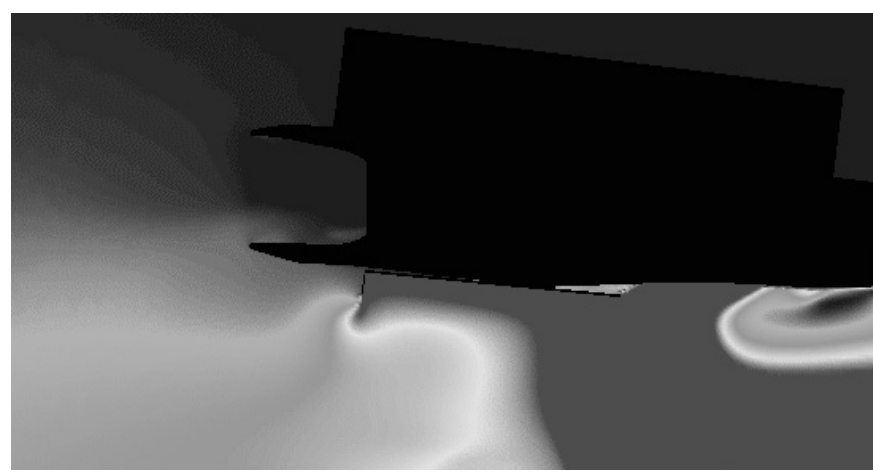

Figure 29. Temperature contours through engine intake in the range $20-60^{\circ} \mathrm{C}$, at time $t=0.4 \mathrm{sec}$.

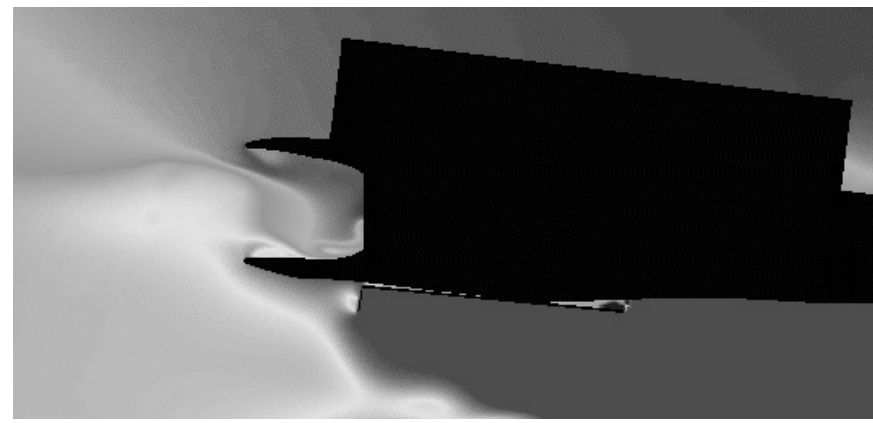

Figure 30 . Temperature contours through engine intake in the range $20-60^{\circ} \mathrm{C}$, at time $t=1.0 \mathrm{sec}$.

the intake, and there appears to be some separation over the leading edge of the intake. The hot-gas ingestion appears mainly on the lower portion of the intake at this particular cut-plane.

All results below (Fig. 22 to Fig. 30) refer to the 'full domain' solution. 


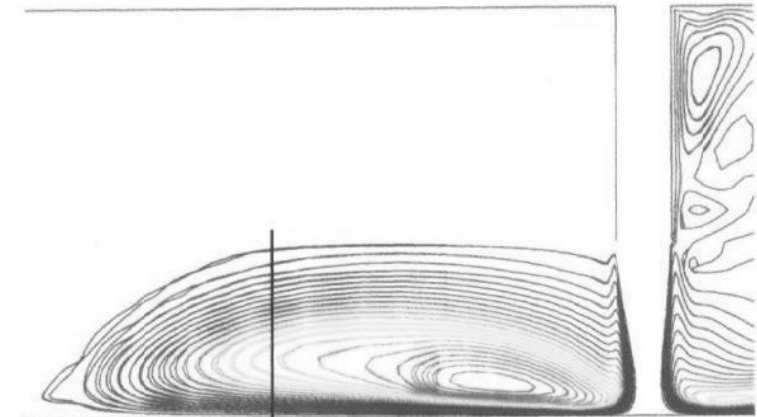

Eddy viscosity in the vortex region for the Spalart-Allmaras turbulence model.

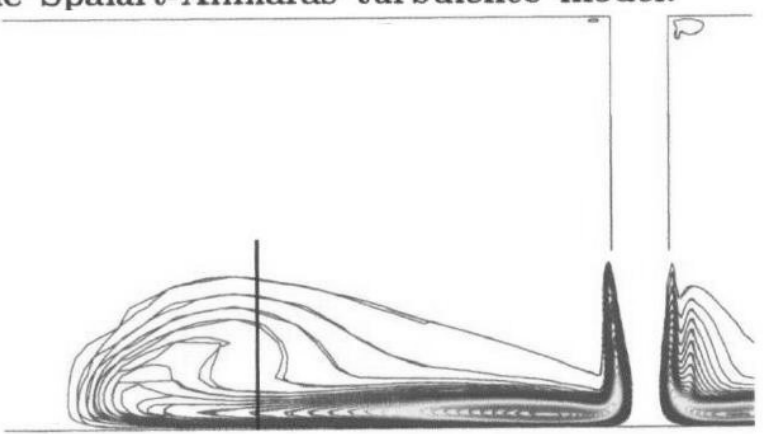

Eddy viscosity in the vortex region for the Spalart-Allmaras turbulence model with a production cutoff.

Figure 31. Ground vortex eddy-viscosity contours ${ }^{(4)}$.

\subsection{CONCLUSIONS}

The objective of the present work was to predict the vertical descent phase of a Harrier aircraft. This has been achieved by development of a linear mesh movement technique based on an existing sinusoidal mesh movement capability within the flow solver. The method allows the user to prescribe a linear translation to a complex $3 \mathrm{D}$ aircraft body, allowing simulation of hover, vertical descent, and landing.

The method has been applied to simulate a hot-gas ingestion testrig experiment, in which a 1/15th scale model of the Pegasus 11-61(408)/AV-8B Harrier aircraft descends and lands, and the temperatures around the engine intake are monitored to assess the severity of HGI. Various meshing strategies have been explored and current results are based on a URANS approach using a hybrid mesh with $22.4 \mathrm{~m}$ cells and $17.3 \mathrm{~m}$ nodes to represent the full flow domain.

This paper demonstrates that it is feasible to use CFD to model an unsteady Harrier model descent, for HGI prediction. The basic physics and effects of dam and strake geometry were captured, even though the precise magnitude and timescale of HGI is not captured.

Simulations of a static twin-jet with intake in cross-flow validation test-case were done. This highlighted some shortcomings of using the Spalart-Allmaras one-equation turbulence model for this type of jet impingement problem. The jet shear layers are too large which causes the potential core to decay too rapidly and this affects the resultant ground layer flow.

For the descending Harrier model test-cases, a comparison of experimental and predicted engine intake temperature profiles indicates that the near field effects have been better predicted than the far-field effects. It is likely this is due to inability of the SA model to predict the full extent of the jet potential core length and resulting ground vortex region.
For the Harrier configuration modelled, the predicted results indicate that the occurrence of HGI is solely due to ingestion of flow from the front two nozzles. In addition, the weaker far-field effects are shown to be responsible for the initial increase in intake temperature profiles, whereas the stronger near-field effects are shown to cause the latter increase in temperatures.

This improved understanding of the HGI mechanisms, could enable the design of potential HGI palliatives such as dams/strakes to be refined/optimised.

It is considered that improved URANS results are obtainable by looking at sensitivity of the solution to turbulence model and inlet boundary layer profile specification. However, LES techniques are required for more accurate representation of the unsteady fluctuating nature of the ingested flows.

Hybrid meshes in which unstructured mesh is used in the nearfield (to capture the complex aircraft geometry) and structured mesh is used for the far-field (as well as key areas of the near-field) are required to provide appropriate mesh resolution for such computations.

We have demonstrated that such large hybrid mesh unsteady computations can be performed on the HPCx supercomputer, but require roughly 25 separate runs on 256 processors for 12 hours at a time, taking about SIX weeks duration.

Issues of visualisation for large data-sets have been addressed in order to post-process the results. A 64-bit version of Fieldview was used with the 'data-guide' function to create animations of the flow properties for the 100 flow solutions.

Virtual reality techniques have been used to study unsteady 3D animations of path-lines, iso-surfaces and surface contours in order to understand how the ground vortex changes shape and location as the aircraft descends. Fieldview ATV software has been used to visualise the transient 3D flow-field.

\subsection{FUTURE WORK}

Sensitivity study for the effects of grid refinement, turbulence model and inlet boundary conditions should be done, in order to obtain the best possible comparison of URANS prediction with experimental results. The turbulence modelling aspect should proceed with (an eddy-viscosity limited) Spalart-Allmaras model and then progress to $k-\varepsilon$ (wall function and low-Re versions). Figure 31 taken from Ref. 4 , shows how the shape of a recirculation region in the ground vortex is directly affected by use of such an eddy-viscosity limiter (which is applied only outside of the boundary layer) within the SA model. LES techniques are to be applied to current URANS solutions that represent the aircraft at touchdown. This will allow some assessment of the improvements that LES may offer compared with the URANS approach.

\section{ACKNOWLEDGMENTS}

The authors wish to acknowledge the following bodies and people: EPSRC/DTI/MoD (who funded the project number NMZB/013); Rolls-Royce (Peter Stow, Leigh Lapworth, Richard Bailey and Tony Ponton); ANSYS (Mark Allen - ICEMCFD support); Surrey University (Nick Hills, for his parallel version of the flow solver and support); Loughborough University (Gary Page, Jim McGuirk and Qinling Li); Daresbury Lab (Andy Sunderland - HPCx support). Many thanks are also due to Caleb Dhanasekaran in the CFD Lab at Cambridge University Engineering Department for the continual hardware and software support throughout the duration of this project. The HPCx computer time was provided through the UK Applied Aerodynamics Consortium (UKAAC) under EPSRC grant GR/S91130/01. 


\section{REFERENCES}

1. Richardson, G.A., Dawes, W.N. and Savill, A.M. Hot gas ingestion modelling for the vertical descent phase of a Harrier, 2005, Paper AIAA-5338-2005, 17th AIAA CFD Conference, Toronto, 6-9 June 2005.

2. Richardson, G.A., Dawes, W.N. and Savill, A.M. CFD analysis of hot gas ingestion mechanisms for the vertical descent phase of a Harrier aircraft, 2006, Paper GT2006-90766, GT2006 ASME Turbo Expo, 8-11 May 2006, Barcelona, Spain.

3. Behrouzi, P. and McGuirk, J.J. Experimental Data for CFD validation of the intake ingestion process in STOVL aircraft, Flow Turbulence and Combustion, 2000, 64, pp 233-251.

4. Pandya, S.A., Murman, S.M. and Sankaran, V. Unsteady computations of a jet in crossflow with ground effect, 2003, AIAA Paper 20033890, 23rd AIAA Fluid Dynamics Conference and Exhibit, 23-26 June 2003, Orlando, FL.

5. Page, G.J., McGuirk, J.J. ET AL Application of computational fluid dynamics to hot gas ingestion modelling, September 1998, International Powered Lift Conference.

6. ChaderJian, N.M. and Pandya, S.A. (NASA Ames), Ahmad, J.U. and Murman, S.M. (ELORET). Progress towards generation of a NavierStokes database for a Harrier in ground effect, AIAA 2002-5966.

7. MoInier, P. and GiLes, M.B. Preconditioned Euler and Navier-Stokes calculations on unstructured grids, 1998, Sixth ICFD Conference on Numerical Methods for Fluid Dynamics, Oxford, UK.

8. Spalart, P.R. and Allmaras, S.R. A one-equation turbulence model for aerodynamic flows, La Recherche Aerospatiale, 1994, 1, pp 5-21.

9. Martinelli, L. Calculations of Viscous Flows with a Multigrid Method, 1987, PhD thesis, Dept of Mech and Aerospace Eng, Princeton University, USA

10. Muller, J.D. and Giles, M.B. Edge-based multigrid schemes for hybrid grids, 1998, Sixth ICFD Conference on Numerical Methods for Fluid Dynamics, Oxford.

11. GILES, M. UNSFLO: a numerical method for the calculation of unsteady flow in turbo machinery, May 1991, GTL Report No 205.

12. Li, Q., PAGe, G.J. and McGuirk, J.J. Large-eddy simulation of twin impinging jets in crossflow, Aeronaut $J, 111,(1117)$, March 2006.

13. Penrose, C.J. Harrier 2 hot gas reingestion model tests, INTERNAL Rolls-Royce (Bristol) Report, GN 30224, August 1990

14. Harper, L.R. Harrier 2 hot gas reingestion model tests, phase II, INTERNAL Rolls-Royce (Bristol) Report, GN 30345, September 1990. 